\title{
Right temporal variant frontotemporal dementia is pathologically heterogeneous: a case-series and a systematic review
}

\author{
Hulya Ulugut ${ }^{*} \mathbb{D}$, Anke A. Dijkstra² ${ }^{2}$, Marta Scarioni ${ }^{1}$, Netherlands Brain Bank ${ }^{3}$, Frederik Barkhof ${ }^{4,5}$, \\ Philip Scheltens ${ }^{1}$, Annemieke J. M. Rozemuller ${ }^{2}$ and Yolande A. L. Pijnenburg ${ }^{1}$
}

\begin{abstract}
Although the right temporal variant frontotemporal dementia (rtvFTD) is characterised by distinct clinical and radiological features, its underlying histopathology remains elusive. Being considered a right-sided variant of semantic variant primary progressive aphasia (SVPPA), TDP-43 type C pathology has been linked to the syndrome, but this has not been studied in detail in large cohorts. In this case report and systematic review, we report the autopsy results of five subjects diagnosed with rtvFTD from our cohort and 44 single rtvFTD subjects from the literature. Macroscopic pathological evaluation of the combined results revealed that rtvFTD demonstrated either a frontotemporal or temporal evolution, even if the degeneration started in the right temporal lobe initially. FTLD-TDP type C was the most common underlying pathology in rtvFTD, however, in 64\% of rtvFTD, other underlying pathologies than FTLD-TDP type $C$ were present, such as Tau-MAPT and FTLD-TDP type A and B. Additionally, accompanying motor neuron or corticospinal tract degeneration was observed in $28 \%$ of rtvFTD patients. Our results show that in contrast to the general assumption, rtvFTD might not be a pure FTLD-TDP type C disorder, unlike its left temporal counterpart svPPA. Large sample size pathological studies are warranted to understand the diverse pathologies of the right and left temporal variants of frontotemporal dementia.
\end{abstract}

Keywords: Dementia, Frontotemporal lobar degeneration, Frontotemporal dementia, Right temporal lobe atrophy, Semantic dementia, Pathology, FTLD-TDP, Tauopathies

\section{Introduction}

Frontotemporal dementia (FTD) is a neurodegenerative disorder that predominantly affects the frontal and/ or temporal lobes. It is subdivided into three different prototypic subtypes; semantic dementia (SD), progressive non-fluent aphasia (PNFA) and behavioural variant frontotemporal dementia (bvFTD) [1]. In 2011, consensus clinical diagnostic criteria were revised and FTD was classified as behavioural variant [2] whereas SD, PNFA

\footnotetext{
*Correspondence: ulugut.hulya@gmail.com

${ }^{1}$ Alzheimer Center Amsterdam, Department of Neurology, Amsterdam

Neuroscience, Vrije Universiteit Amsterdam, Amsterdam UMC, De Boelelaan 1118, 1081 HZ Amsterdam, The Netherlands

Full list of author information is available at the end of the article
}

and logopenic variant primary progressive aphasia (PPA) were classified under the umbrella of PPA [3]. On the other hand, a number of studies reported a separate syndromic variant that predominantly affects the right temporal lobe (rtvFTD), usually accompanied by behavioural changes, memory deficit and prosopagnosia [4-9]. While rtvFTD cannot formally be considered a PPA variant due to the absence of aphasia, there have been reports of rtvFTD presenting with non-verbal semantic deficits[10] and neuro-radiological studies have shown mirror image findings, suggesting that they might reflect the same pathophysiological process, albeit on opposite sides [3, 11-13].

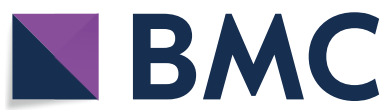

(c) The Author(s) 2021. Open Access This article is licensed under a Creative Commons Attribution 4.0 International License, which permits use, sharing, adaptation, distribution and reproduction in any medium or format, as long as you give appropriate credit to the original author(s) and the source, provide a link to the Creative Commons licence, and indicate if changes were made. The images or other third party material in this article are included in the article's Creative Commons licence, unless indicated otherwise in a credit line to the material. If material is not included in the article's Creative Commons licence and your intended use is not permitted by statutory regulation or exceeds the permitted use, you will need to obtain permission directly from the copyright holder. To view a copy of this licence, visit http://creativecommons.org/licenses/by/4.0/. The Creative Commons Public Domain Dedication waiver (http://creativeco mmons.org/publicdomain/zero/1.0/) applies to the data made available in this article, unless otherwise stated in a credit line to the data. 
Pathological examination plays a key role in understanding the nature of the diseases. Unsurprisingly, the neuropathology underlying clinical FTD is also heterogeneous [14]. The term frontotemporal lobar degeneration (FTLD) is used to encompass pathological conditions that present as clinical FTD. FTLD has been classified into four main groups based on the major proteins accumulation in the brain: tau protein (FTLD-tau); TAR DNA-binding protein 43 (FTLD-TDP); ubiquitin positive, TDP-43 negative and immunopositive for the fused in sarcoma protein (FTLD-FUS); and a remaining group encompassing the few cases characterized by inclusions that label only for markers of the ubiquitin proteasome system (FTLD-UPS) or no inclusions [15]. Based on the morphology and cortical distribution of the accumulation, the two main groups (FTLD-tau and FTLD-TDP) have been subdivided; Pick's disease (PiD), corticobasal degeneration (CBD), progressive supranuclear palsy (PSP), argyrophilic grain disease (AGD), globular glial tauopathy (GGT) and FTD caused by microtubule association protein tau (MAPT) for FTLD-tau [15-18] and the subtypes A, B, C, D and E for FTLD-TDP [19]. These pathological subgroups and their specific pathologies are linked to a number of clinical syndromes. Whereas clinico-pathological concordance is generally weak, particularly for bvFTD, a strong clinicopathological concordance with the underlying FTLD-TDP type $C$ pathology is present in SvPPA [20-22].

Since rtvFTD is sometimes considered a type of svPPA $[3,11,12]$, FTLD-TDP type C pathology has been linked to the syndrome [13]. Recently, we have described the different clinical progression patterns of rtvFTD and svPPA [9], leading to the question whether their underlying pathologies may differ. To our knowledge, only one post-mortem study has focused on the pathological characteristics of rtvFTD, highlighting the possible association of rtvFTD with underlying tau-pathology [7]. Therefore, we aimed to determine the range of FTLD molecular pathologies underlying the clinical syndrome of rtvFTD based on a combination of clinico-pathological data from the Amsterdam Dementia Cohort and a review of the literature.

\section{Methods}

\section{Patient selection}

We identified all subjects diagnosed with FTD and/ or PPA from the Amsterdam Dementia Cohort [23] recruited between 1994 and $2019(n=669)$ who had a pathological confirmation of their clinical diagnosis $(\mathrm{n}=32)$ (Ethical approval protocol no: 2016.061). From this group, patients were selected who had a predominant right temporal lobar atrophy on the initial neuroimaging $(n=5)$ (Fig. 1). In all rtvFTD subjects, the atrophy scores of the right temporal lobe [24-26] were higher (at least 1 grade) than the left temporal lobe and the frontal lobes, as assessed by an experienced neuroradiologist, blinded to the clinical diagnosis (FB). The visual rating scores are displayed in the results section (Table 1). Additionally, in our sample, the frontal atrophy scores were less than grade-1[25] and none of the subjects met the diagnostic criteria of svPPA [3], while all fulfilled at least 2 symptoms out of prosopagnosia, episodic memory impairment, and behavioural change [9], and their clinical profile was in line with the previously reported rtvFTD case series $[4,7,8]$ (Fig. 1). Additionally, isolated right temporal lobar hypo-perfusion was reported in Case 1 on perfusion SPECT and isolated right temporal hypometabolism in Case 3 on FDG-PET imaging, in other centres before being referred to us.

\section{Clinical and neuropsychological assessment}

All 5 rtvFTD subjects had been followed throughout their disease course by an experienced behavioural neurologist. The case notes of all rtvFTD subjects were scrutinized retrospectively. All initial and annual follow-up reports were reviewed by a senior behavioural neurologist (Y.P.) blinded to pathological information. Initial clinical symptoms were collected and family history of any neurodegenerative or psychiatric disease was recorded. The emergence of motor deficits (pyramidal or extrapyramidal) and progression to different clinical syndromes over the disease course was recorded. The following data were extracted of all subjects at the time of initial visit: Clinical Dementia Rating Scale (CDR) [27] and Mini Mental State Examination (MMSE) [28] as global measures, episodic memory [visual association test (VAT) A [29] and the Dutch version of the Rey Auditory Verbal Learning Test (RAVLT)] [30], executive functions [Frontal assessment Battery (FAB) [31], trail making test (TMT) B [32] and digit span backward [33]], language [VAT naming [29]], attention [digit span forward [33] and TMT A [32]] and visuospatial functions [Visual Objective and Space Perception (VOSP)- Dot counting [34]].

\section{Neuropathological analysis}

Subjects were included from the Netherlands Brain Bank and department of pathology, Amsterdam UMC, location Vumc, where tissue was collected according to the local legal and ethical guidelines. All histological slides were re-examined according to the current classification system (A.A.D.) $[15,19]$. All pathological examinations were conducted by an expert neuropathologist (A.R.) The pattern of FTLD-TDP pathology was classified into the five following subtypes; A, B, C, D and E $[19,35]$. The pattern of FTLD-tau pathology was classified into the 


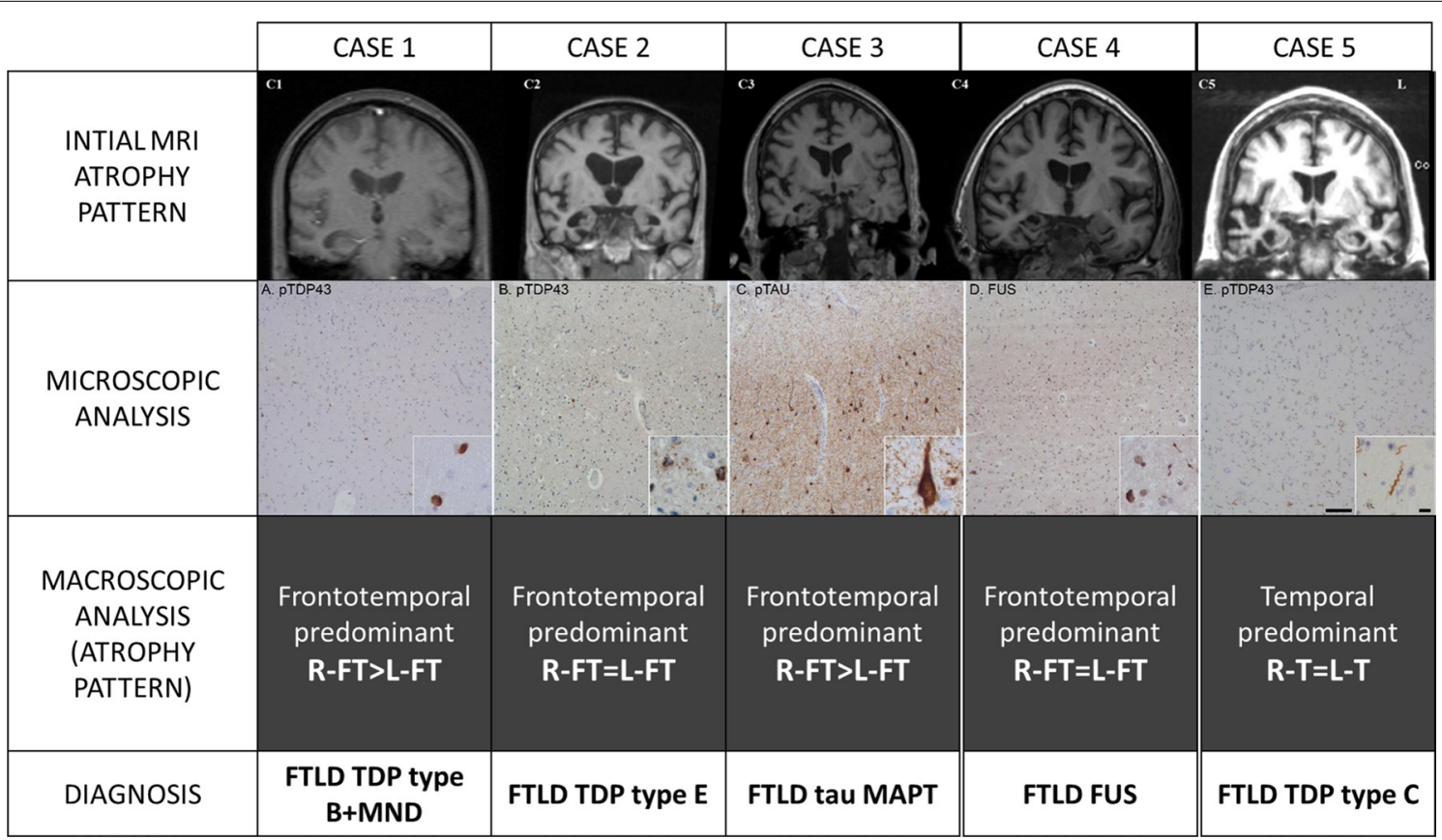

Fig. 1 Different pathological diagnoses in donors with rtvFTD. The cases with rtvFTD displayed pathology from different pathological molecular subclasses in FTD. Although all pathological accumulations started from the right temporal lobe, according to the initial MRI atrophy pattern, over time the patients exhibited heterogeneous progression patterns. Case 1 showed FTD-TDP-B pathology with predominant neuronal inclusions throughout the cortical layers in right temporal lobe (a). Clinically, motor neuron disease developed over the disease course. Case 2 showed FTD-TDP-E pathology characterised by granulofilamentous neuronal inclusions (insert) and grains in right temporal lobe (b). The pathology spread to bilateral fronto-temporal areas. Clinically, this was accompanied by severe behavioural and language problems. Case 3 had tau-pathology with threads and tangles and some plaques (Anterior cingulate cortex: C, adapted from Ulugut Erkoyun et al.,2021, JAD, CC BY-NC 4.0). At the end stage of the disease, right predominant frontotemporal atrophy was observed based on the macroscopic pathological examination. The clinical evolution involved the development of atypical Parkinsonism. Case 4 had large FUS-positive neuronal inclusions and FUS-positive threads (D: right frontal lobe), developed severe global atrophy at a clinical picture of becoming mutistic and bedridden in 4 years after diagnosis. Lastly, case 5 showed long dystrophic neurites characteristic for FTD-TDP-C (E: insular cortex) and developed bilateral temporal atrophy at the end stage of the disease, based on the pathological examination. This patient's clinical features were relatively benign, presenting with verbal and non-verbal semantic impairment and without the development of any motor disturbances and a disease duration of 12 years. Scalebar is $100 \mu m$, scalebar insert is $10 \mu \mathrm{m}$

six following categories; PiD, PSP, CBD, GGT, AGD and FTD caused by MAPT mutations (tau-MAPT) $[15,18]$. Co-existing pathological features such as Alzheimer's disease (AD) [36], Motor neuron degeneration (MND) [37], corticospinal tract degeneration (CTD) [38] and dementia with Lewy bodies (DLB) [39] were recorded.

Details of the pathological examination are presented in Additional file 1.

\section{Systematic review}

We conducted a systematic review following PRISMA guidelines [40] to identify the papers reporting pathological features of rtvFTD patients with available clinical and neuroimaging data (Additional file 2). The search was completed in December 2019 on two electronic databases; Pubmed and Embase. The following terms were used for the search: ("frontotemporal lobar degeneration"
OR "frontotemporal dementia" OR "right temporal " OR "semantic dementia") AND ("pathology") NOT ("epilepsy" OR "tumor"). No filter was employed in the search. Titles and abstracts of the papers were screened according to the following eligibility criteria:

1. Original research, including case series and individual case reports.

2. Exclusion of review articles and animal studies.

3. Exclusion of reports with insufficient information.

Disagreements on eligibility were resolved through discussion among the authors (Additional file 3).

After detailed screening, 34 studies were eligible for systematic review. Patients with the following diagnoses "right temporal variant FTD", "right temporal variant semantic dementia", "right temporal variant svPPA", 
Table 1 Reclassification of the reported molecular neuropathologies

\begin{tabular}{|c|c|c|c|c|}
\hline & Publications & $\mathrm{N}$ & Reported molecular neuropathology & Adapted diagnosis \\
\hline 1 & [52] & 1 & FTLD-TDP type $C+C T D$ & FTLD-TDP type $C+C T D$ \\
\hline 2 & [69] & 1 & FTLD-tau-PSP + TDP type A & FTLD-tau-PSP + TDP type A \\
\hline 3 & [54] & 9 & $\begin{array}{l}\text { FTLD-tau-PiD }(n=1) \\
\text { FTLD-TDP type } C(n=8)\end{array}$ & $\begin{array}{l}\text { FTLD-tau-PiD }(n=1) \\
\text { FTLD-TDP type } C(n=8)\end{array}$ \\
\hline 4 & [61] & 1 & FTLD-TDP type $\mathrm{C}+$ tau-PSP & FTLD-TDP type $\mathrm{C}+$ tau-PSP \\
\hline 5 & [64] & 1 & FTLD-TDP type A & FTLD-TDP type A \\
\hline 6 & [63] & 1 & FTLD-TDP type A & FTLD-TDP type A \\
\hline 7 & [70] & 1 & FTLD-tau-PiD & FTLD-tau-PiD \\
\hline 8 & [67] & 1 & $\begin{array}{l}\text { TDP-43 pathology in all cortical layers. NCl, with crescentic, round, skein-like and granular } \\
\text { types. Short threads accompanied the NCI. Due to the admixture of neuronal cytoplas- } \\
\text { mic inclusion subtypes seen in FTLD-TDP type A and type B, presence of type A threads, } \\
\text { but involvement of all cortical layers (type B), the pattern of TDP- } 43 \text { inclusions is unclas- } \\
\text { sifiable. Skein-like inclusions in lower motor neurons, producing the neuropathological } \\
\text { diagnosis of motor neuron disease. Thal amyloid plaque stage 4, Braak 1. 4R-only atypical } \\
\text { tauopathy }\end{array}$ & FTLD-TDP type A-B + AD + 4R tau \\
\hline 9 & [55] & 1 & FTLD-tau-GGT & FTLD-tau-GGT \\
\hline 10 & [38] & 7 & $\begin{array}{l}\text { FTLD-TDP type } C(n=1) \\
\text { FTLD-TDP type } C+C T D(n=6)\end{array}$ & $\begin{array}{l}\text { FTLD-TDP type } C(n=1) \\
\text { FTLD-TDP type } C+C T D(n=6)\end{array}$ \\
\hline 11 & [56] & 2 & $\begin{array}{l}\text { FTLD-TDP Mackenzie type } 3+\operatorname{MND}(n=1) \\
\text { TDP Mackenzie type } 3+\text { MND }+ \text { AD }(n=1)\end{array}$ & $\begin{array}{l}\text { FTLD TDP type } B+M N D^{*} \\
\text { FTLD TDP type } B+M N D^{*}+A D\end{array}$ \\
\hline 12 & [66] & 1 & FTLD-FUS & FTLD-FUS \\
\hline 13 & [68] & 1 & FTLD-TDP Mackenzie type 3+ MND & FTLD-TDP type B + MND* \\
\hline 14 & [60] & 1 & FTLD-TDP-43 pathology with NII & FTLD-TDP type A-B \\
\hline 15 & [62] & 1 & FTLD-TDP Cairns type 2+ MND & FTLD-TDP type B + MND* \\
\hline 16 & {$[65]$} & 1 & $\begin{array}{l}\text { Immunohistochemistry using antibodies to ubiquitin showed } \mathrm{NCls} \text {, some of these inclu- } \\
\text { sions were also immunoreactive for phosphorylated TDP- } 43 \text { antibodies. We identified no } \\
\text { DN, but a few NCl, which were positive for both ubiquitin and phosphorylated TDP-43 }\end{array}$ & FTLD-TDP type A-B + MND* \\
\hline 17 & [4] & 1 & Mixed Alzheimer and cortical Lewy body disease $(n=1)$ & $A D+D L B(n=1)$ \\
\hline 18 & [38] & 8 & $\begin{array}{l}\text { FTLD-tau-PiD }(n=1) \\
\text { FTLD-tau-MAPT }(n=7)\end{array}$ & $\begin{array}{l}\text { FTLD-tau-PiD }(n=1) \\
\text { FTLD-tau-MAPT }(n=7)\end{array}$ \\
\hline 19 & [59] & 1 & TDP-43 pathology with NII & FTLD-TDP type A-B \\
\hline 20 & {$[71]$} & 1 & $\begin{array}{l}\text { Tau-negative, TDP-43-positive neuronal cytoplasmic inclusions and dystrophic neurites } \\
\text { were found. Numerous NFTs and senile plaques with amyloid angiopathy indicated } \\
\text { advanced Alzheimer disease }\end{array}$ & FTLD-TDP type A-B + AD \\
\hline 21 & [58] & 1 & TDP43 pathology with NII + MND & FTLD-TDP type A-B + MND* \\
\hline
\end{tabular}

TDP: TAR DNA-binding protein 43; TAU: tau protein; MND: motor neuron disease; MAPT: microtubule associated protein; FUS: fused in sarcoma protein; PiD: Pick's disease; PSP: progressive supranuclear palsy; FTLD-U: frontotemporal lobar degeneration with tau-negative, ubiquitin-immunoreactive pathology; AD: Alzheimer's disease; DLB: dementia with Lewy bodies; NII: neuronal cytoplasmic and intranuclear inclusions

${ }^{*}$ : Clinically diagnosed with MND

"bvFTD presenting with right temporal atrophy", "right temporal variant bvFTD", "FTD patient with right temporal atrophy", "right predominant semantic dementia" were included. Therefore, non-FTD clinical diagnoses such as amyotrophic lateral sclerosis (ALS) or atypical Parkinsonism were excluded. Of note, all case notes and neuroimaging features were also re-assessed. If the left temporal or frontal atrophy was equal or higher than the right temporal atrophy, the subjects were not included. In all included studies, the atrophy pattern had been assessed with either unbiased standardized volumetric morphometry analysis or visual scoring scales. Additionally, in all studies, neuroimaging had been displayed that allowed us to re-assess the radiological features. If detailed radiological information was not eligible, those studies were considered as articles with insufficient information and excluded. Lastly, the clinical features of all cases had been in line with the published rtvFTD literature $[4,8,9]$. Furthermore, all studies were examined in detail to remove cases without TDP-43 staining and when case duplication occurred in, we selected the study from a particular institution/ cohort over a given period of time with the largest sample size. Thirteen studies were excluded based on the criteria mentioned above following author consensus (Additional file 3). This yielded a sample of 21 studies $(n=44)$ which have defined the 
molecular pathology in the patients with predominant right temporal atrophy and a consistent clinical syndrome $[4,8,9]$ (Table 2). The data from all 44 subjects were combined with our 5 rtvFTD subjects to analyse clinico-pathological associations in rtvFTD.

Since the classification of the molecular neuropathology of FTD has been updated over the years, we adapted all reviewed pathology reports based on the current classification system and the subtype nomenclature used was that of the more recent harmonized classification system; FTLD-TDP type A=Mackenzie type 1/Sampathu type 3 , type $B=$ Mackenzie type $3 /$ Sampathu type 2 , type $\mathrm{C}=$ Mackenzie type $2 /$ Sampathu type 1 , type $\mathrm{D}=$ type 4 with VCP mutations $[15,35]$. There was no correction for the FTLD-TDP type E diagnosis [19]. In a subset of cases, however available pathological data were insufficient to identify either TDP type A or B. These cases were denominated as TDP-A-B (Table 2).

\section{Results}

\section{Demographic and clinical data of our cohort}

All Amsterdam cases were right-handed. The rtvFTD group comprised 4 male and 1 female patients. Demographic data, detailed clinical symptoms and cognitive test results are displayed in Table 1 . All subjects had behavioural problems, depression and memory deficits. While 3 of them had prosopagnosia, 4 of them had word finding difficulties. Additionally, they became negativistic, non-flexible, sensitive to pain, very fixated on certain thoughts or activities, and they lost their logical reasoning. For instance, due to drinking while driving, Case 2's driver's license was withdrawn, which means that he could no longer be a volunteer for the Red Cross. Interestingly, while he did not care for his driving licence, he became obsessed with working in the Red Cross. On the other hand, Case 3 decided to be the golf champion in the Netherlands and spent his entire time and money for this sport, even though he became extremely stingy regarding other daily life activities, including costs for showering. Other cases also displayed bizarre rituals such as walking/cycling for miles in the same route every day or repeating the same eating/drinking routine etc. Change of personal taste (food, colours, music etc.) was another prominent feature. Importantly, their behavioural profile was quite different from bvFTD [2], and they had several non-verbal semantic deficits that might cause those behavioural-psychiatric problems. Furthermore, unlike svPPA, aphasia was not the most prominent feature and neither svPPA diagnostic criteria covered their initial symptom distribution [3], however their clinical phenotypes were in line with the published rtvFTD literature $[4,8,9]$. Although rtvFTD cases had fairly similar initial clinical presentations, over the years, they exhibited a different progression pattern. While the clinical diagnosis of three of the cases remained FTD, one of the cases (Case 1) developed concomitant MND, whereas another patient carrying a heterozygous Ser352Leu mutation in the MAPT gene developed atypical parkinsonism (Case 3) (Table 1). The underlying genetics of this case have been published recently [41].

\section{Pathological features of our cohort}

Details of the pathological results of the Amsterdam subjects are displayed in Table 3. The rtvFTD group exhibited a heterogeneous underlying pathology, including FTLD- TDP type B with motor neuron degeneration, FTLD-TDP type E, FTLD- MAPT, FTLD-FUS, and FTLD-TDP type C (Fig. 1). The macroscopic analysis revealed that except Case 5, who had an underlying TDP- C pathology and a predominant bilateral temporal atrophy, all rtvFTD cases had either right predominant or bilateral frontotemporal involvement at the end stage of the disease. (Table 4).

\section{Systematic review}

The pathological data of 21 studies from 13 centres could be pooled and various molecular neuropathological associations were observed (Table 5). The combination of our results and the results of the systematic review revealed that the underlying pathology of $\operatorname{rtvFTD}(n=49)$ was heterogeneous (Fig. 2). The two most common underlying pathologies in rtvFTD were FTLD-TDP (67.3\%) and FTLD-tau (26.5\%). The observed FTLD-TDP subtypes were FTLD-TDP type C (36.7\%), type B (10.2\%), type A (4.1\%), type $\mathrm{E}(2 \%)$, whereas $16.3 \%$ of cases were labelled as FTLD-TDP type A-B. Despite the relatively high frequency of FTLD-TDP type $C$ pathology, 7 out of 18 FTLD-TDP type $C$ subjects had a CTD co-pathology and one subject diagnosed with FTLD-TDP type $C$ had a tau-PSP co-pathology. In other FTLD-TDP sub-groups, co-pathologies such as MND, tau and AD also occurred (Fig. 2). The observed FTLD-tau subtypes were tauMAPT (16.3\%), tau-PiD (6.1\%), tau-GGT (2\%) and tauPSP (2\%). The minority of the subjects was diagnosed with FTLD-FUS (4.1\%) and only one subject had concomitant AD and DLB pathology.

Macroscopic findings were reported in 14 out of 21 studies. The combination of our results and the literature $(n=25)$ revealed that the macroscopic atrophy pattern was again heterogeneous in rtvFTD. Frontotemporal predominant involvement was reported in 11 out of 25 subjects whereas 14 exhibited a temporal predominant atrophy pattern. One FUS case had a striatal predominant atrophy pattern alongside frontotemporal atrophy. Whereas 8 out of 9 TDP type $C$ cases had temporal predominant atrophy in the macroscopic examination, other 
Table 2 Initial clinical features of the rtvFTD subjects

\begin{tabular}{|c|c|c|c|c|c|}
\hline & Case 1 & Case 2 & Case 3 & Case 4 & Case 5 \\
\hline Age & 58 & 68 & 59 & 59 & 63 \\
\hline Sex & Male & Male & Male & Male & Female \\
\hline Handedness & Right & Right & Right & Right & Right \\
\hline \multicolumn{6}{|l|}{ Symptoms } \\
\hline Prosopagnosia & $\sqrt{ }$ & & $\sqrt{ }$ & & $\sqrt{ }$ \\
\hline Memory deficit & $\sqrt{ }$ & $\sqrt{ }$ & $\sqrt{ }$ & $\sqrt{ }$ & $\sqrt{ }$ \\
\hline Disinhibition & $\sqrt{ }$ & $\sqrt{ }$ & $\sqrt{ }$ & $\sqrt{ }$ & \\
\hline Apathy-inertia & $\sqrt{ }$ & $\sqrt{ }$ & $\sqrt{ }$ & $\sqrt{ }$ & $\sqrt{ }$ \\
\hline Alexithymia & & & $\sqrt{ }$ & $\sqrt{ }$ & $\sqrt{ }$ \\
\hline Bizarre preoccupations & $\sqrt{ }$ & $\sqrt{ }$ & $\sqrt{ }$ & $\sqrt{ }$ & $\sqrt{ }$ \\
\hline $\begin{array}{l}\text { Lack of logical reason- } \\
\text { ing }\end{array}$ & $\sqrt{ }$ & $\sqrt{ }$ & $\sqrt{ }$ & $\sqrt{ }$ & $\sqrt{ }$ \\
\hline $\begin{array}{l}\text { Pathological dwelling } \\
\text { on one activity }\end{array}$ & $\sqrt{ }$ & $\sqrt{ }$ & $\sqrt{ }$ & $\sqrt{ }$ & $\sqrt{ }$ \\
\hline $\begin{array}{l}\text { Change of personal } \\
\text { taste }\end{array}$ & & $\sqrt{ }$ & $\sqrt{ }$ & & \\
\hline Nicotine/alcohol abuse & & $\sqrt{ }$ & & & \\
\hline Hyperalgesia & & & $\sqrt{ }$ & $\sqrt{ }$ & \\
\hline $\begin{array}{l}\text { Over sleeping during } \\
\text { the day }\end{array}$ & $\sqrt{ }$ & & & $\sqrt{ }$ & \\
\hline $\begin{array}{l}\text { Word finding difficul- } \\
\text { ties }\end{array}$ & $\sqrt{ }$ & $\sqrt{ }$ & & $\sqrt{ }$ & $\sqrt{ }$ \\
\hline Naming difficulties & $\sqrt{ }$ & & & & $\sqrt{ }$ \\
\hline $\begin{array}{l}\text { Single word compre- } \\
\text { hension deficit }\end{array}$ & & & & & $\sqrt{ }$ \\
\hline Depression & $\sqrt{ }$ & $\sqrt{ }$ & $\sqrt{ }$ & $\sqrt{ }$ & $\sqrt{ }$ \\
\hline Slowness & $\sqrt{ }$ & $\sqrt{ }$ & $\sqrt{ }$ & $\sqrt{ }$ & \\
\hline Motor restless & & & & $\sqrt{ }$ & \\
\hline \multicolumn{6}{|l|}{ Hyper-orality } \\
\hline $\begin{array}{l}\text { Diagnosis prior to } \\
\text { autopsy }\end{array}$ & $\mathrm{FTD}+\mathrm{MND}$ & FTD & $\begin{array}{l}\text { FTD + atypical parkin- } \\
\text { sonism }\end{array}$ & FTD & FTD \\
\hline Family History & $\begin{array}{l}\text { Father had psychiatric } \\
\text { symptoms, sister had } \\
\text { paranoid disorder }\end{array}$ & $\begin{array}{l}\text { Two brothers and } \\
\text { mother had dementia } \\
\text { at the age of } 70 \text { s with } \\
\text { behavioural problems }\end{array}$ & $\begin{array}{l}\text { Mother had psychiat- } \\
\text { ric symptoms and } \\
\text { attempt a suicide, } \\
\text { uncle (maternal) had } \\
\text { dementia at the age } \\
\text { of } 85\end{array}$ & $\begin{array}{l}\text { Father and brother had } \\
\text { depression, son had } \\
\text { ADHD }\end{array}$ & $\begin{array}{l}\text { Mother attempt a } \\
\text { suicide }\end{array}$ \\
\hline $\begin{array}{l}\text { MRI anterior temporal } \\
\text { R/L }\end{array}$ & $2 / 0$ & $3 / 2$ & $2 / 0$ & $3 / 1$ & $4 / 3$ \\
\hline MRI mesial temporal R/L & $3 / 0$ & $4 / 3$ & $2 / 1$ & $4 / 0$ & $3 / 2$ \\
\hline MRI frontal R/L & $1 / 0$ & $1 / 1$ & $1 / 0$ & $1 / 1$ & $1 / 0$ \\
\hline SPECT/PET & $\begin{array}{l}\text { Right temporal hypo- } \\
\text { perfusion }\end{array}$ & N.A & $\begin{array}{l}\text { Right temporal hypo- } \\
\text { perfusion }\end{array}$ & N.A & N.A \\
\hline Genetic analysis & N.A & N.A & $\begin{array}{l}\text { MAPT }(+) \\
\text { Ser352Leu }\end{array}$ & N.A & $\begin{array}{l}\text { MAPT (negative) } \\
\text { PRGN (negative) } \\
\text { C9orf72 (negative) }\end{array}$ \\
\hline CDR & 0.5 & 0.5 & 0.5 & 0.5 & 1 \\
\hline MMSE & $27 / 30$ & $25 / 30$ & $23 / 30$ & $22 / 30$ & $25 / 30$ \\
\hline FAB & N.A & N.A & $14 / 18$ & 18/18 & $14 / 18$ \\
\hline VAT-A & N.A & $6 / 12$ & $4 / 12$ & $4 / 12$ & $7 / 12$ \\
\hline RAVLT delayed recall & N.A & $0 / 15$ & N.A & $12 / 15$ & N.A \\
\hline VAT naming & N.A & N.A & $10 / 12$ & $12 / 12$ & $6 / 12$ \\
\hline Digit span forward & N.A & N.A & $12 / 16$ & $13 / 16$ & $8 / 16$ \\
\hline
\end{tabular}


Table 2 (continued)

\begin{tabular}{llllll}
\hline & Case 1 & Case 2 & Case 3 & Case 4 & Case 5 \\
\hline Digit span backward & N.A & N.A & $8 / 16$ & $7 / 16$ & $8 / 16$ \\
TMT A & N.A & N.A & $57^{\prime \prime}(\mathrm{A})$ & $69^{\prime \prime}(\mathrm{A})$ & $49^{\prime \prime}(\mathrm{A})$ \\
TMT B & N.A & N.A & $169^{\prime \prime}(\mathrm{LA})$ & $166^{\prime \prime}(\mathrm{LA})$ & $102^{\prime \prime}(\mathrm{A})$ \\
VOSP-Dot Counting & N.A & N.A & $10 / 10$ & $8 / 10$ & $9 / 10$ \\
\hline
\end{tabular}

rtvFTD: right temporal variant frontotemporal dementia; bvFTD: behavioural variant frontotemporal dementia; svPPA: semantic variant primary progressive aphasia; MND: motor neuron disease; ADHD: attention deficit hyperactivity disorder; FTD; frontotemporal dementia, CDR; Clinical dementia rating, MMSE; mini-mental state examination, VAT; visual association test, RAVLT; Dutch version of the Rey Auditory Verbal Learning Test, FAB; frontal assessment battery, TMT; trial making test, VOSP; Visual objective and space perception, A; Avarage, LA; low average, N.A.; not available

Table 3 Pathological features of rtvFTD cases

\begin{tabular}{|c|c|c|c|c|c|}
\hline & Case 1 & Case 2 & Case 3 & Case 4 & Case 5 \\
\hline \multicolumn{6}{|l|}{ Macroscopic analysis } \\
\hline Brain weight & $1117 \mathrm{gr}$ & $1410 \mathrm{gr}$ & $1260 \mathrm{gr}$ & $1010 \mathrm{gr}$ & $975 \mathrm{gr}$ \\
\hline Atrophy & FT-Right & $\mathrm{FT}$ & FT-Right & $\mathrm{FT}$ & $\mathrm{T}$ \\
\hline Substantia nigra & Normally pigmented & Normally pigmented & Slightly pale & Pale & Slightly pale \\
\hline Locus coeruleus & Visible & Visible & Right < Left & Not visible & Visible \\
\hline Atherosclerosis & No & Moderate & Severe & Mild & No \\
\hline \multicolumn{6}{|l|}{ Microscopic analysis } \\
\hline Plaque and tangles & Negative & Negative & Thal 3 & Negative & Negative \\
\hline Congo red & Negative & Negative & Negative & Negative & Negative \\
\hline Alpha synuclein & Negative & Negative & Negative & Braak 3 & Negative \\
\hline Tau & Negative & Negative & Positive & Negative & Negative \\
\hline Pick Bodies & No & No & No & No & No \\
\hline TDP-43 & Positive & Positive & Negative & Negative & Positive \\
\hline FUS & Negative & Negative & Negative & Positive & Negative \\
\hline Accumulation & All layers & Predominantly layer 2 & $3 R+4 R$ & FUS & Several long threads \\
\hline Frontal & ++ & ++ & +++ & +++ & + \\
\hline Temporal & +++ & +++ & $+++^{*}$ & +++ & +++ \\
\hline Motor cortex & +++ & - & - & $\mathrm{n} / \mathrm{a}$ & - \\
\hline Corticospinal tract & +++ & - & - & - & - \\
\hline Parietal & - & - & ++ & $\mathrm{n} / \mathrm{a}$ & + \\
\hline Occipital & - & - & + & - & - \\
\hline Hippocampus & +++ & +++ & +++ & +++ & +++ \\
\hline Amygdala & +++ & +++ & +++ & +++ & +++ \\
\hline Caudate, putamen & - & ++ & +++ & +++ & ++ \\
\hline Thalamus & - & ++ & ++ & +++ & ++ \\
\hline Brain stem & - & - & +++ & +++ & - \\
\hline Cerebellum & - & - & - & - & - \\
\hline Cervical cord & +++ & - & - & - & - \\
\hline Diagnosis & FTLD-TDP type B + MND & FTLD-TDP type E & FTLD-MAPT & FTLD-FUS & FTLD-TDP type C \\
\hline
\end{tabular}

rtvFTD: Right temporal variant frontotemporal dementia, FTLD: Frontotemporal lobar degeneration, MAPT: Microtubule associated protein tau, TDP-43: TAR DNAbinding protein 43, n/a: not available, F: Frontal, T: Temporal, R: Repeat

*Extensive tau positivity indicates a primary tauopathy. Pathological results are suggestive for tau mutation

+++ : Severe, ++: Moderate, +: Mild, -: Normal

subtypes such as FTLD-tau or TDP type A-B had either temporal predominant atrophy at the end stage of the disease. Of note, macroscopic atrophy results were available only in 4 tau and 9 TDP type A or B cases (Fig. 3).

\section{Discussion}

In this case series and systematic review, we ascertained the heterogeneous underlying molecular neuropathology of rtvFTD, showing that it cannot be considered a 
Table 4 Pathological features of diagnostic groups

\begin{tabular}{llll}
\hline Case & Diagnosis & Macroscopic analysis (atrophy pattern) & Microscopic analysis \\
\hline 1 & rtvFTD & Frontotemporal predominant R-FT > L-FT & FTLD-TDP type B+ MND \\
2 & rtvFTD & Frontotemporal predominant R-FT $=$ L-FT & FTLD-TDP type E \\
3 & rtvFTD & Frontotemporal predominant R-FT > L-FT & FTLD-tau-MAPT \\
4 & rtvFTD & Frontotemporal predominant R-FT $=$ L-FT & FTLD-FUS \\
5 & rtvFTD & Temporal predominant R-T $=$ L-T & FTLD-TDP type C
\end{tabular}

rtvFTD: right temporal variant frontotemporal dementia; sVPPA: semantic variant primary progressive aphasia; R: right; L: left; F: frontal; T: temporal; TDP: TAR DNAbinding protein 43; TAU: tau protein; MND: motor neuron disease; MAPT: microtubule associated protein; FUS: fused in sarcoma protein; PiD: Pick's disease

Table 5 Outcomes of the included studies

\begin{tabular}{|c|c|c|c|c|c|c|}
\hline & Publications & $\mathbf{N}$ & Institution & Country & Macroscopy (atrophy pattern) & Microscopy \\
\hline 1 & {$[52]$} & 1 & UCL & UK & Frontotemporal predominant R-FT $=\mathrm{L}-\mathrm{FT}$ & TDP type $C+C T D$ \\
\hline 2 & {$[69]$} & 1 & MCCN & UK & Temporal predominant $\mathrm{R}-\mathrm{T}=\mathrm{L}-\mathrm{T}$ & Tau-PSP + TDP type A \\
\hline 3 & {$[54]$} & 9 & UCSF & USA & $\begin{array}{l}\text { Frontotemporal predominant R-FT }=\mathrm{L}-\mathrm{FT}(\mathrm{n}=1) \\
\text { TDP-C: N.A }\end{array}$ & $\begin{array}{l}\text { Tau-PiD }(n=1) \\
\text { TDP type } C(n=8)\end{array}$ \\
\hline 4 & {$[61]$} & 1 & UCSF & USA & N.A & TDP type $C+$ Tau-PSP \\
\hline 5 & {$[63]$} & 1 & $\mathrm{UCL}$ & UK & Temporal predominant $\mathrm{R}-\mathrm{T}=\mathrm{L}-\mathrm{T}$ & TDP type A \\
\hline 6 & {$[64]$} & 1 & Helsinki University & Finland & Frontotemporal predominant R-FT $=\mathrm{L}-\mathrm{FT}$ & TDP type A \\
\hline 7 & {$[70]$} & 1 & Cambridge Brain Bank & UK & Temporal predominant $\mathrm{R}-\mathrm{T}=\mathrm{L}-\mathrm{T}$ & Tau-MAPT \\
\hline 8 & {$[55]$} & 1 & UCL & UK & Frontotemporal predominant R-FT > L-FT & Tau-GGT \\
\hline 9 & {$[67]$} & 1 & UCSF & USA & Frontotemporal predominant R-FT > L-FT & TDP type $A-B+A D+4 R$ tau \\
\hline 10 & {$[38]$} & 7 & Mayo Clinic & USA & Individual data N.A. Overall, temporal predominant & $\begin{array}{l}\text { TDP type } C(n=1) \\
\text { TDP type } C+\operatorname{CTD}(n=6)\end{array}$ \\
\hline 11 & {$[56]$} & 2 & Mayo Clinic & USA & N.A & $\begin{array}{l}\text { TDP type } B+M N D^{*}(n=1) \\
\text { TDP type } B+M N D^{*}+A D(n=1)\end{array}$ \\
\hline 12 & {$[66]$} & 1 & UCSF & USA & Striatal predominant & FUS \\
\hline 13 & {$[68]$} & 1 & Uppsala University & Sweden & Temporal predominant $\mathrm{R}-\mathrm{T}>\mathrm{L}-\mathrm{T}$ & TDP type B + MND* \\
\hline 14 & {$[60]$} & 1 & $\mathrm{UCL}$ & UK & N.A & TDP type A-B \\
\hline 15 & {$[62]$} & 1 & Tokyo IP & Japan & Temporal predominant $\mathrm{R}-\mathrm{T}>\mathrm{L}-\mathrm{T}$ & TDP type $B+M N D^{*}$ \\
\hline 16 & {$[65]$} & 1 & Tokyo IP & Japan & Temporal predominant $\mathrm{R}-\mathrm{T}>\mathrm{L}-\mathrm{T}$ & TDP type $A-B+M N D^{*}$ \\
\hline 17 & {$[4]$} & 1 & UCL & UK & N.A & $A D+D L B(n=1)$ \\
\hline 18 & {$[38]$} & 8 & Mayo clinic & USA & N.A & $\begin{array}{l}\text { Tau-PiD }(n=1) \\
\text { Tau-MAPT }(n=7)\end{array}$ \\
\hline 19 & {$[59]$} & 2 & $\mathrm{UCL}$ & UK & N.A & TDP type A-B $(n=2)$ \\
\hline 20 & {$[71]$} & 1 & Aichi University & Japan & Frontotemporal predominant $\mathrm{R}-\mathrm{FT}=\mathrm{L}-\mathrm{FT}$ & TDP type $A-B+A D$ \\
\hline 21 & {$[58]$} & 1 & $\begin{array}{l}\text { Northwestern } \\
\text { University }\end{array}$ & USA & N.A & TDP type $A-B+M N D^{*}$ \\
\hline
\end{tabular}

TDP: TAR DNA-binding protein 43; TAU: tau protein; CTD: corticospinal tract degeneration; MND: motor neuron disease; MAPT: microtubule associated protein; FUS: fused in sarcoma protein; PiD: Pick's disease; PSP: progressive supranuclear palsy; FTLD-U: frontotemporal lobar degeneration with tau-negative, ubiquitinimmunoreactive pathology; AD: Alzheimer's disease; DLB: dementia with Lewy bodies; UCL: University College London; UCSF: University of California San Francisco; FTD: frontotemporal dementia; MCCN: Manchester Centre for Clinical Neurosciences; IP: institute of psychiatry; VAPSHCS: Veterans Affairs Puget Sound Health Care System

*Clinically diagnosed with FTD + MND

pure FTLD-TDP type C syndrome. In rtvFTD, the most common underlying pathologies were FTLD-TDP type $\mathrm{C}$, tau-MAPT as well as TDP type A and B, whereas its left temporal counterpart; svPPA links to the TDP type $\mathrm{C}$ pathology. Moreover, accompanying MND or CTD was prominent in rtvFTD, whereas this has not been reported in larger studies on svPPA [20-22].
Furthermore, the macroscopic descriptions revealed that although neurodegeneration started in the right temporal lobe according to initial neuroimaging, atrophy spread to either the frontal areas or left temporal area which might be the explanation of the heterogeneous clinical progression pattern in rtvFTD. 


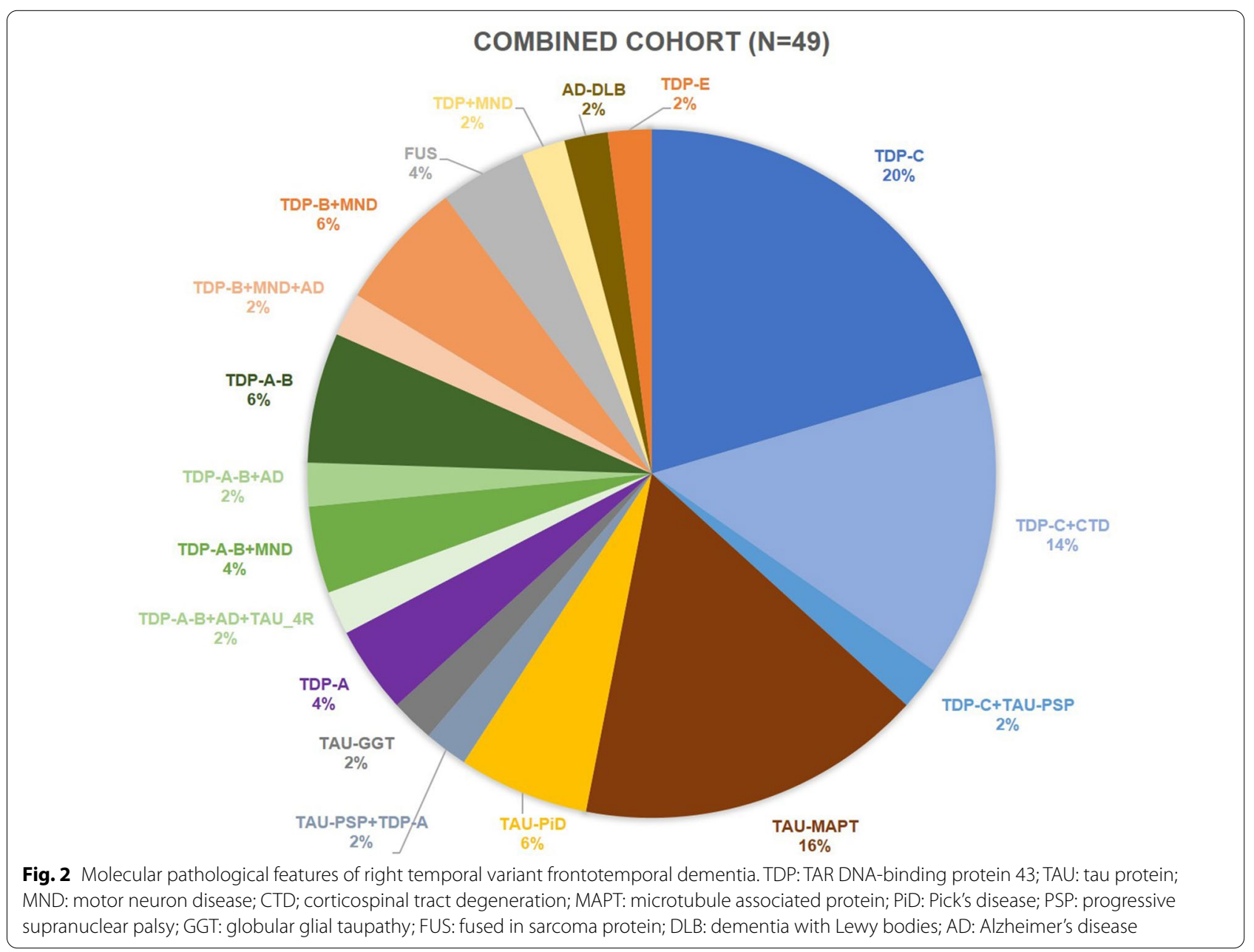

The systematic review showed that TDP type $C$ pathology was the most common underlying pathology of rtvFTD. Still in the combined dataset, it was observed in only a third of rtvFTD patients and approximately half of them had a co-pathology such as CTD and tau-PSP.

Following the FTLD-TDP type $C$ diagnosis, the second most common pathological diagnosis of rtvFTD was FTLD-MAPT. This result might be expected because the association between tau mutations and anterior temporal atrophy is well known [42-45] and genetic studies have shown the relationship between tau mutations and rtvFTD [7, 41]. However, the relationship between specific right temporal atrophy and tau mutations is still unknown. According to previous studies, FTLD-MAPT exhibits a symmetrical atrophy pattern, despite the fact that clinically, the most common tau mutations produce behavioural symptoms and later semantic impairment $[42,44]$ which resembles the clinical profile of rtvFTD [9]. Additionally, the association between svPPA and MAPT mutations is quite rare [22, 46-48]. Moreover, a recent GENFI paper reported that in the pre-symptomatic carriers of the MAPT, GRN and C9orf72 genes, there was significant evidence of atrophy in the right anterior insula and they suggested that there may be some distinct regions in which the disease process starts [49]. This may explain the pathological diversity between two temporal lobe disorders; rtvFTD and svPPA. Future studies combining neurodevelopmental, embryonic, clinical, genetic and pathological findings will be required to further understand the biological basis of selective and lateralized neurodegeneration.

It has previously been suggested that rtvFTD can be divided into two major subtypes; the semantic clinical phenotype associated with temporal atrophy and TDP type $C$ pathology and the behavioural type associated with frontal atrophy and FTLD-MAPT [7]. Even though our study confirms the observation of two anatomical rtvFTD variants, we argue that the motor component of the syndrome should not be neglected. However, due to low case numbers, we cannot derive associations with specific types of underlying pathology. Future larger dataset studies are warranted to elucidate the underlying 


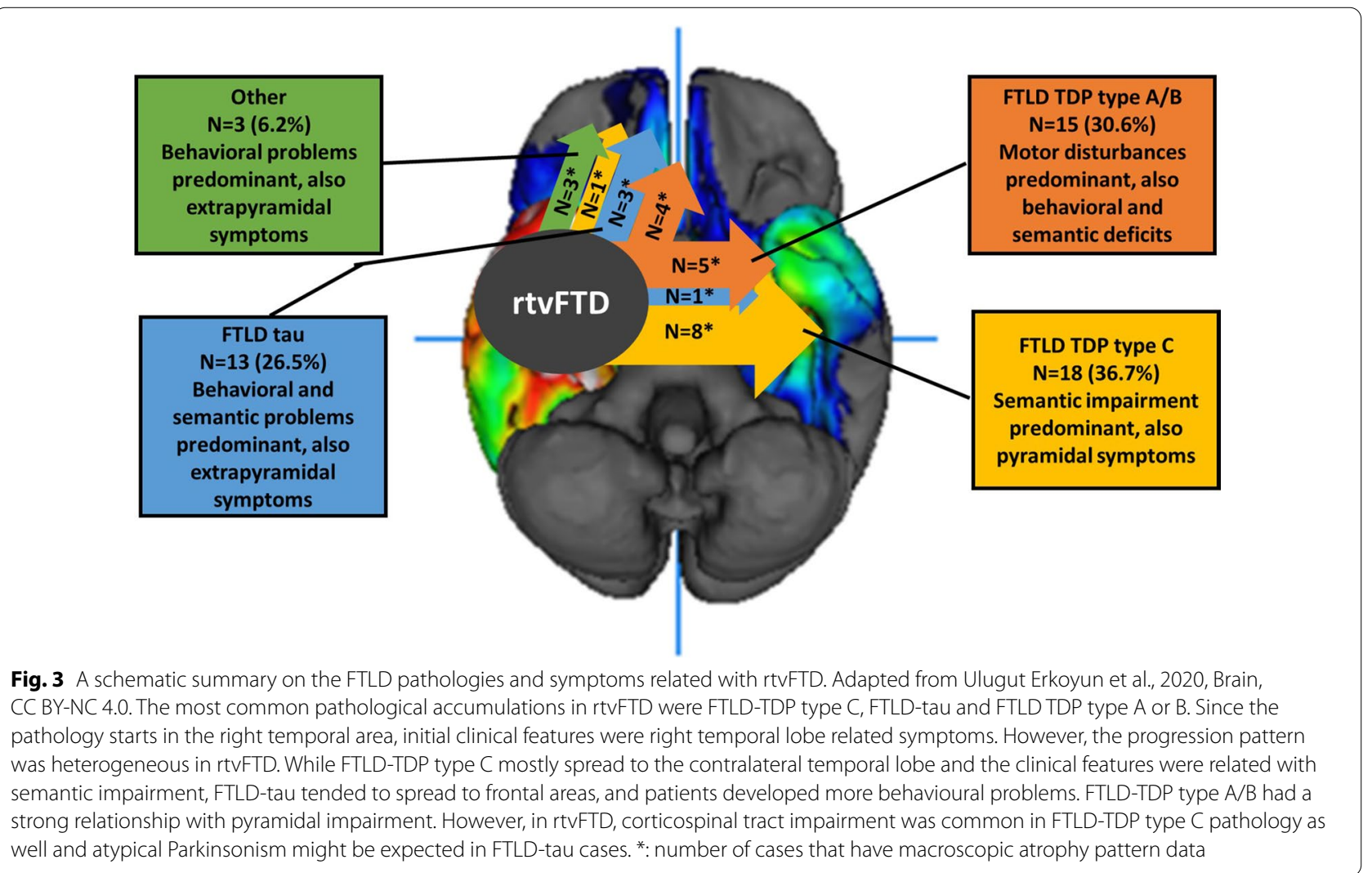

pathology specific clinical presentation and progression pattern in rtvFTD.

In contrast to the previous argument, Borghesani et al., (2020) suggested that the left and the right temporal variant of FTD should be considered the same disease based on their similar neuroanatomical progression patterns within the temporal and contralateral temporal regions[50]. However, the limitation of that study is that only subjects with TDP type C pathology were included, thereby potentially excluding other underlying pathologies with a different progression pattern. Additionally, it must be noted that most neuropathological studies taking into account the underlying neuropathology of rtvFTD were based on svPPA cohorts[20,50], hence reports of underlying pathology of rtvFTD diagnosed with bvFTD are lacking.

One of the important results of our study is the relationship between rtvFTD and co-existing MND or CTD features. Co-existing CTD or MND was observed in $28.6 \%$ of rtvFTD subjects in our combined dataset. The general assumption is that ALS links to either bvFTD or nfvPPA while the association with svPPA is rare [51]. In addition, although previous pathological studies have revealed that those accompanying pathologies are mostly related with either FTLD-TDP type B or A- B subtypes
[35], our results point out that CTD might accompany FTLD-TDP type C, in particular in rtvFTD. This association was also suggested by Josephs and colleagues (2013) [7]. Of note, some authors have reported the combination of left predominant temporal atrophy and CTD [52, 53]. Recently, we described the clinical profile of rtvFTD and reported that slowness is a distinctive symptom of rtvFTD in particular in the later stages of the disease [9]. Underlying tau pathology and MND form a potential explanation of this clinical observation.

One of our cases was found to harbour FTLD-FUS pathology. Consistent with the literature [15], FUS pathology is rare, and we show that the phenotype can also present as rtvFTD. In addition, another rtvFTD subject was diagnosed with FTLD-TDP type $E$ in our cohort. TDP type $E$ has been recently identified based on a small number of case series, and links to prominent behavioural and movement disturbances that was also consistent with our case [19].

This is the first study that systematically collected the underlying molecular neuropathology of rtvFTD, which challenges the assumption that rtvFTD is an FTLDTDP type $C$ disorder by reporting heterogeneous FTLD pathologies in the patients with rtvFTD. However, there are some limitations that need to be addressed. 
First of all, the number of our subjects was limited and the results mostly rely on the literature review. Secondly, current neuropathological criteria for FTLD could not be applied in all rtvFTD cases described in the literature.

The right temporal lobe plays a key role in memory, social cognition, verbal and especially non-verbal semantic cognition. Therefore, rtvFTD can present with a combination of psychiatric features and multi-domain cognitive impairment. Our results show that heterogeneous FTLD pathologies can initially cause right temporal lobe neurodegeneration and present with rtvFTD clinical features. To date, due to the lack of separate diagnostic criteria, rtvFTD has been relatively neglected in the large clinicopathological studies, although our findings of highly heterogeneous underlying pathologies in rtvFTD might have consequences for individualised patient management. Predominant semantic impairment associated with the predominant temporal lobe atrophy is related with the FTLD-TDP type C pathology whereas FTLD-tau is mostly related with behavioural problems and frontotemporal atrophy at the later stages of the disorder. Pyramidal and extrapyramidal disturbances are expected in rtvFTD not only in patients with FTLD-TDP type A/B, but also in FTLD-TDP type $C$ and tau.

Our findings suggest that rtvFTD might be a separate pathological entity and future large scale studies are warranted to shed light on whether the presentation, disease course and associated pathology provide the evidence for this.

\begin{abstract}
Abbreviations
AD: Alzheimer's disease; AGD: Argyrophilic grain disease; ALS: Amyotrophic lateral sclerosis; bvFTD: Behavioural variant frontotemporal dementia; CBD: Corticobasal degeneration; FTD: Frontotemporal dementia; FTLD: Frontotemporal lobar degeneration; FUS: Fused in sarcoma; GGT: Globular glial tauopathy; MAPT: Microtubule associated protein tau; MND: Motor neuron degeneration; nfvPPA: Nonfluent variant primary progressive aphasia; PNFA: Progressive nonfluent aphasia; PSP: Progressive supranuclear palsy; PPA: Primary progressive aphasia; rtVFTD: Right temporal variant frontotemporal dementia; SD: Semantic dementia; sVPPA: Semantic variant primary progressive aphasia; TDP-43: TAR DNA binding protein 43.
\end{abstract}

\section{Supplementary Information}

The online version contains supplementary material available at https://doi. org/10.1186/s40478-021-01229-z.

Additional file 1. Details of the pathological examination.

Additional file 2. PRISMA flow diagram.

Additional file 3. Excluded studies.

\section{Authors' contributions}

Conception and design of the study: HU, YP and PS Radiological assessment: FB Pathological assessment: AR, AAD and MS Acquisition and analysis of data: all authors. Drafting manuscript: $\mathrm{HU}$ and YP Figures: $\mathrm{HU}$ and AAD. All authors read and approved the final manuscript.

\section{Funding}

Research of the Alzheimer Centre Amsterdam is part of the neurodegeneration research program of Amsterdam Neuroscience. The Alzheimer Centre Amsterdam is supported by Stichting Alzheimer Nederland and Stichting VUmc fonds. FB is supported by the NIHR biomedical research centre at UCLH. Yolande A.L. Pijnenburg is recipient of the JPND-funded project (FTD-DIPPA).

\section{Availability of data and materials}

Data are available on request from the corresponding author.

\section{Declarations}

\section{Ethics approval and consent to participate}

The local Medical Ethics Committee approved a general protocol for using the clinical and pathological data for research purposes. (Ethical approval protocol No.: 2016.061).

\section{Consent for publication}

Not applicable.

\section{Competing interests}

The authors declare that they have no competing interests.

\section{Author details}

${ }^{1}$ Alzheimer Center Amsterdam, Department of Neurology, Amsterdam Neuroscience, Vrije Universiteit Amsterdam, Amsterdam UMC, De Boelelaan 1118, $1081 \mathrm{HZ}$ Amsterdam, The Netherlands. ${ }^{2}$ Department of Pathology, Vrije Universiteit Amsterdam, Amsterdam UMC, Amsterdam, The Netherlands. ${ }^{3}$ Netherlands Institute for Neuroscience, Amsterdam, The Netherlands. ${ }^{4}$ Department of Radiology and Nuclear Medicine, Vrije Universiteit Amsterdam, Amsterdam UMC, Amsterdam, The Netherlands. ${ }^{5}$ UCL Institutes of Neurology and Healthcare Engineering, University College London, London, UK.

Received: 15 June 2021 Accepted: 12 July 2021

Published online: 03 August 2021

\section{References}

1. Neary D, Snowden JS, Gustafson L, Passant U, Stuss D, Black S, Freedman M, Kertesz A, Robert PH, Albert M, Boone K, Miller BL, Cummings J, Benson DF (1998) Frontotemporal lobar degeneration: a consensus on clinical diagnostic criteria. Neurology 51:1546-1554

2. Rascovsky K, Hodges JR, Knopman D, Mendez MF, Kramer JH, Neuhaus J, van Swieten JC, Seelaar H, Dopper EG, Onyike CU, Hillis AE, Josephs KA, Boeve BF, Kertesz A, Seeley WW, Rankin KP, Johnson JK, Gorno-Tempini ML, Rosen H, Prioleau-Latham CE, Lee A, Kipps CM, Lillo P, Piguet O, Rohrer JD, Rossor MN, Warren JD, Fox NC, Galasko D, Salmon DP, Black SE, Mesulam M, Weintraub S, Dickerson BC, Diehl-Schmid J, Pasquier F, Deramecourt V, Lebert F, Pijnenburg Y, Chow TW, Manes F, Grafman J, Cappa SF, Freedman M, Grossman M, Miller BL (2011) Sensitivity of revised diagnostic criteria for the behavioural variant of frontotemporal dementia. Brain 134:2456-2477

3. Gorno-Tempini ML, Hillis AE, Weintraub S, Kertesz A, Mendez M, Cappa SF, Ogar JM, Rohrer JD, Black S, Boeve BF, Manes F, Dronkers NF, Vandenberghe R, Rascovsky K, Patterson K, Miller BL, Knopman DS, Hodges JR, Mesulam MM, Grossman M (2011) Classification of primary progressive aphasia and its variants. Neurology 76:1006-1014

4. Chan D, Anderson V, Pijnenburg Y, Whitwell J, Barnes J, Scahill R, Stevens JM, Barkhof F, Scheltens P, Rossor MN, Fox NC (2009) The clinical profile of right temporal lobe atrophy. Brain 132:1287-1298

5. Kumfor F, Landin-Romero R, Devenney E, Hutchings R, Grasso R, Hodges JR, Piguet O (2016) On the right side? A longitudinal study of left-versus right-lateralized semantic dementia. Brain 139:986-998

6. Pozueta A, Lage C, Garcia-Martinez M, Kazimierczak M, Bravo M, LopezGarcia S, Riancho J, Gonzalez-Suarez A, Vazquez-Higuera JL, de ArcochaTorres M, Banzo I, Jimenez-Bonilla J, Berciano J, Rodriguez-Rodriguez E, Sanchez-Juan P (2019) Cognitive and behavioral profiles of left and right semantic dementia: differential diagnosis with behavioral variant frontotemporal dementia and Alzheimer's disease. J Alzheimers Dis 
7. Josephs KA, Whitwell JL, Knopman DS, Boeve BF, Vemuri P, Senjem ML, Parisi JE, Ivnik RJ, Dickson DW, Petersen RC, Jack CR Jr (2009) Two distinct subtypes of right temporal variant frontotemporal dementia. Neurology 73:1443-1450

8. Thompson SA, Patterson K, Hodges JR (2003) Left/right asymmetry of atrophy in semantic dementia: behavioral-cognitive implications. Neurology 61:1196-1203

9. Ulugut Erkoyun H, Groot C, Heilbron R, Nelissen A, van Rossum J, Jutten R, Koene T, van der Flier WM, Wattjes MP, Scheltens P, Ossenkoppele R, Barkhof F, Pijnenburg Y (2020) A clinical-radiological framework of the right temporal variant of frontotemporal dementia. Brain

10. Snowden JS, Harris JM, Thompson JC, Kobylecki C, Jones M, Richardson AM, Neary D (2018) Semantic dementia and the left and right temporal lobes. Cortex 107:188-203

11. Seeley WW, Bauer AM, Miller BL, Gorno-Tempini ML, Kramer JH, Weiner M, Rosen HJ (2005) The natural history of temporal variant frontotemporal dementia. Neurology 64:1384-1390

12. Brambati SM, Rankin KP, Narvid J, Seeley WW, Dean D, Rosen HJ, Miller BL, Ashburner J, Gorno-Tempini ML (2009) Atrophy progression in semantic dementia with asymmetric temporal involvement: a tensor-based morphometry study. Neurobiol Aging 30:103-111

13. Rohrer JD, Geser F, Zhou J, Gennatas ED, Sidhu M, Trojanowski JQ, Dearmond SJ, Miller BL, Seeley WW (2010) TDP-43 subtypes are associated with distinct atrophy patterns in frontotemporal dementia. Neurology 75:2204-2211

14. Cairns NJ, Bigio EH, Mackenzie IR, Neumann M, Lee VM, Hatanpaa KJ, White CL 3rd, Schneider JA, Grinberg LT, Halliday G, Duyckaerts C, Lowe JS, Holm IE, Tolnay M, Okamoto K, Yokoo H, Murayama S, Woulfe J, Munoz DG, Dickson DW, Ince PG, Trojanowski JQ, Mann DM, Consortium for Frontotemporal Lobar D (2007) Neuropathologic diagnostic and nosologic criteria for frontotemporal lobar degeneration: consensus of the Consortium for Frontotemporal Lobar Degeneration. Acta Neuropathol 114:5-22

15. Mackenzie IR, Neumann M (2016) Molecular neuropathology of frontotemporal dementia: insights into disease mechanisms from postmortem studies. J Neurochem 138(Suppl 1):54-70

16. Lee G, Leugers CJ (2012) Tau and tauopathies. Prog Mol Biol Transl Sci 107:263-293

17. Lee VM, Goedert M, Trojanowski JQ (2001) Neurodegenerative tauopathies. Annu Rev Neurosci 24:1121-1159

18. Kovacs GG (2015) Invited review: neuropathology of tauopathies: principles and practice. Neuropathol Appl Neurobiol 41:3-23

19. Lee EB, Porta S, Michael Baer G, Xu Y, Suh E, Kwong LK, Elman L, Grossman M, Lee VM, Irwin DJ, Van Deerlin VM, Trojanowski JQ (2017) Expansion of the classification of FTLD-TDP: distinct pathology associated with rapidly progressive frontotemporal degeneration. Acta Neuropathol 134:65-78

20. Hodges JR, Mitchell J, Dawson K, Spillantini MG, Xuereb JH, McMonagle P, Nestor PJ, Patterson K (2010) Semantic dementia: demography, familial factors and survival in a consecutive series of 100 cases. Brain 133:300-306

21. Spinelli EG, Mandelli ML, Miller ZA, Santos-Santos MA, Wilson SM, Agosta F, Grinberg LT, Huang EJ, Trojanowski JQ, Meyer M, Henry ML, Comi G, Rabinovici G, Rosen HJ, Filippi M, Miller BL, Seeley WW, Gorno-Tempin ML (2017) Typical and atypical pathology in primary progressive aphasia variants. Ann Neurol 81:430-443

22. Rohrer JD, Lashley T, Schott JM, Warren JE, Mead S, Isaacs AM, Beck J, Hardy J, de Silva R, Warrington E, Troakes C, Al-Sarraj S, King A, Borroni B, Clarkson MJ, Ourselin S, Holton JL, Fox NC, Revesz T, Rossor MN, Warren JD (2011) Clinical and neuroanatomical signatures of tissue pathology in frontotemporal lobar degeneration. Brain 134:2565-2581

23. van der Flier WM, Pijnenburg YA, Prins N, Lemstra AW, Bouwman FH, Teunissen CE, van Berckel BN, Stam CJ, Barkhof F, Visser PJ, van Egmond E, Scheltens P (2014) Optimizing patient care and research: the Amsterdam Dementia Cohort. J Alzheimers Dis 41:313-327

24. Scheltens $P$, Leys D, Barkhof F, Huglo D, Weinstein HC, Vermersch P, Kuiper M, Steinling M, Wolters EC, Valk J (1992) Atrophy of medial temporal lobes on MRI in "probable" Alzheimer's disease and normal ageing: diagnostic value and neuropsychological correlates. J Neurol Neurosurg Psychiatry 55:967-972

25. Kipps CM, Davies RR, Mitchell J, Kril JJ, Halliday GM, Hodges JR (2007) Clinical significance of lobar atrophy in frontotemporal dementia: application of an MRI visual rating scale. Dement Geriatr Cogn Disord 23:334-342

26. Harper L, Barkhof F, Fox NC, Schott JM (2015) Using visual rating to diagnose dementia: a critical evaluation of MRI atrophy scales. J Neurol Neurosurg Psychiatry 86:1225-1233

27. Morris JC (1993) The Clinical Dementia Rating (CDR): current version and scoring rules. Neurology 43:2412-2414

28. Folstein MF, Folstein SE, McHugh PR (1975) "Mini-mental state". A practical method for grading the cognitive state of patients for the clinician. J Psychiatr Res 12:189-198

29. Lindeboom J, Schmand B, Tulner L, Walstra G, Jonker C (2002) Visual association test to detect early dementia of the Alzheimer type. J Neurol Neurosurg Psychiatry 73:126-133

30. Schmidt (1996) Rey auditory verbal learning test: A handbook. Western Psychological Services Los Angeles.

31. Dubois B, Slachevsky A, Litvan I, Pillon B (2000) The FAB: a Frontal Assessment Battery at bedside. Neurology 55:1621-1626

32. Tombaugh TN (2004) Trail Making Test A and B: normative data stratified by age and education. Arch Clin Neuropsychol 19:203-214

33. Wechsler (2008) Wechsler adult intelligence scale-Fourth Edition (WAIS-IV). San Antonio, TX NCS Pearson

34. Quental NB, Brucki SM, Bueno OF (2013) Visuospatial function in early Alzheimer's disease-the use of the Visual Object and Space Perception (VOSP) battery. PLOS ONE 8:e68398

35. Mackenzie IR, Neumann M (2017) Reappraisal of TDP-43 pathology in FTLD-U subtypes. Acta Neuropathol 134:79-96

36. Braak H, Thal DR, Ghebremedhin E, Del Tredici K (2011) Stages of the pathologic process in Alzheimer disease: age categories from 1 to 100 years. J Neuropathol Exp Neurol 70:960-969

37. Saberi S, Stauffer JE, Schulte DJ, Ravits J (2015) Neuropathology of amyotrophic lateral sclerosis and its variants. Neurol Clin 33:855-876

38. Josephs KA, Whitwell JL, Murray ME, Parisi JE, Graff-Radford NR, Knopman DS, Boeve BF, Senjem ML, Rademakers R, Jack CR Jr, Petersen RC, Dickson DW (2013) Corticospinal tract degeneration associated with TDP-43 type C pathology and semantic dementia. Brain 136:455-470

39. Fujishiro H, Ferman TJ, Boeve BF, Smith GE, Graff-Radford NR, Uitti RJ, Wszolek ZK, Knopman DS, Petersen RC, Parisi JE, Dickson DW (2008) Validation of the neuropathologic criteria of the third consortium for dementia with Lewy bodies for prospectively diagnosed cases. J Neuropathol Exp Neurol 67:649-656

40. Moher D, Liberati A, Tetzlaff J, Altman DG, Group P (2009) Preferred reporting items for systematic reviews and meta-analyses: the PRISMA statement. PLoS Med 6:e1000097

41. Ulugut Erkoyun $H$, van der Lee SJ, Nijmeijer B, van Spaendonk R, Nelissen A, Scarioni M, Dijkstra A, Samancı B, Gürvit H, Yıldırım Z, Tepgeç F, Bilgic B, Barkhof F, Rozemuller A, van der Flier WM, Scheltens P, CohnHokke P, Pijnenburg Y (2021) The right temporal variant of frontotemporal dementia is not genetically sporadic: a case series. J Alzheimers Dis 79:1195-1201

42. Rohrer JD, Ridgway GR, Modat M, Ourselin S, Mead S, Fox NC, Rossor MN, Warren JD (2010) Distinct profiles of brain atrophy in frontotemporal lobar degeneration caused by progranulin and tau mutations. Neuroimage 53:1070-1076

43. Whitwell JL, Jack CR Jr, Boeve BF, Senjem ML, Baker M, Ivnik RJ, Knopman DS, Wszolek ZK, Petersen RC, Rademakers R, Josephs KA (2009) Atrophy patterns in IVS10+16, IVS10+3, N279K, S305N, P301L, and V337M MAPT mutations. Neurology 73:1058-1065

44. Whitwell JL, Jack CR Jr, Boeve BF, Senjem ML, Baker M, Rademakers R, Ivnik RJ, Knopman DS, Wszolek ZK, Petersen RC, Josephs KA (2009) Voxel-based morphometry patterns of atrophy in FTLD with mutations in MAPT or PGRN. Neurology 72:813-820

45. Whitwell $\mathrm{J}$, Josephs KA (2012) Recent advances in the imaging of frontotemporal dementia. Curr Neurol Neurosci Rep 12:715-723

46. Landin-Romero R, Tan R, Hodges JR, Kumfor F (2016) An update on semantic dementia: genetics, imaging, and pathology. Alzheimers Res Ther 8:52

47. Rohrer JD, Schott JM (2011) Primary progressive aphasia: defining genetic and pathological subtypes. Curr Alzheimer Res 8:266-272

48. Josephs KA, Hodges JR, Snowden JS, Mackenzie IR, Neumann M, Mann DM, Dickson DW (2011) Neuropathological background of phenotypical variability in frontotemporal dementia. Acta Neuropathol 122:137-153 
49. Cash DM, Bocchetta M, Thomas DL, Dick KM, van Swieten JC, Borroni B, Galimberti D, Masellis M, Tartaglia MC, Rowe JB, Graff C, Tagliavini F, Frisoni GB, Laforce R Jr, Finger E, de Mendonça A, Sorbi S, Rossor MN, Ourselin S, Rohrer JD, Genetic Ftd Initiative G (2018) Patterns of gray matter atrophy in genetic frontotemporal dementia: results from the GENFI study. Neurobiol Aging 62:191-196

50. Borghesani V, Battistella G, Mandelli ML, Welch A, Weis E, Younes K, Neuhaus J, Grinberg LT, Seeley WM, Spina S, Miller B, Miller Z, Gorno-Tempini ML (2020) Regional and hemispheric susceptibility of the temporal lobe to FTLD-TDP type C pathology. Neurolmage Clinical 28:102369

51. Woollacott IOC, Rohrer JD (2016) The clinical spectrum of sporadic and familial forms of frontotemporal dementia. J Neurochem 138:6-31

52. Miki Y, Ling H, Crampsie S, Mummery CJ, Rohrer JD, Jaunmuktane Z, Lashley T, Holton JL (2019) Corticospinal tract degeneration and temporal lobe atrophy in frontotemporal lobar degeneration TDP-43 type C pathology. Neuropathol Appl Neurobiol

53. Yokota O, Tsuchiya K, Arai T, Yagishita S, Matsubara O, Mochizuki A, Tamaoka A, Kawamura M, Yoshida H, Terada S, Ishizu H, Kuroda S, Akiyama $H$ (2009) Clinicopathological characterization of Pick's disease versus frontotemporal lobar degeneration with ubiquitin/TDP-43-positive inclusions. Acta Neuropathol 117:429-444

54. Snowden JS, Kobylecki C, Jones M, Thompson JC, Richardson AM, Mann DMA (2019) Association between semantic dementia and progressive supranuclear palsy. J Neurol Neurosurg Psychiatry 90(1):115-117

55. Caplan A, Marx G, Elofson J, Lis C, Grinberg L, Miller B et al (2018) A case of semantic variant primary progressive aphasia with Pick's pathology. Neurocase 24(2):90-94

56. Kim EJ, Brown JA, Deng J, Hwang JL, Spina S, Miller ZA et al (2018) Mixed TDP-43 proteinopathy and tauopathy in frontotemporal lobar degeneration: nine case series. J Neurol 265(12):2960-2971

57. Kuuluvainen L, Poyhonen M, Pasanen P, Siitonen M, Rummukainen J, Tienari PJ et al (2017) A Novel Loss-of-Function GRN Mutation p.(Tyr229*): Clinical and Neuropathological Features. J Alzheimers Dis 55(3): 1167-74.

58. Koriath CA, Bocchetta M, Brotherhood E, Woollacott IO, Norsworthy P, Simon-Sanchez J et al (2017) The clinical, neuroanatomical, and neuropathologic phenotype of TBK1-associated frontotemporal dementia: a longitudinal case report. Alzheimers Dement (Amst) 6:75-81

59. Wood R, Moodley K, Hodges JR, Allinson K, Spillantini MG, Chan D (2016) Slowly progressive behavioural presentation in two UK cases with the R406W MAPT mutation. Neuropathol Appl Neurobiol 42(3):291-295

60. Moreno F, Rabinovici GD, Karydas A, Miller Z, Hsu SC, Legati A et al (2015) A novel mutation P112H in the TARDBP gene associated with frontotemporal lobar degeneration without motor neuron disease and abundant neuritic amyloid plaques. Acta Neuropathol Commun 3:19

61. Clark CN, Lashley T, Mahoney CJ, Warren JD, Revesz T, Rohrer JD (2015) Temporal variant frontotemporal dementia is associated with globular glial tauopathy. Cogn Behav Neurol 28(2):92-97

62. Coon EA, Whitwell JL, Parisi JE, Dickson DW, Josephs KA (2012) Right temporal variant frontotemporal dementia with motor neuron disease. J Clin Neurosci 19(1):85-91

63. Lee SE, Seeley WW, Poorzand P, Rademakers R, Karydas A, Stanley CM et al (2012) Clinical characterization of bvFTD due to FUS neuropathology. Neurocase 18(4):305-317

64. Ostberg P, Bogdanovic N (2011) Semantic dementia with lower motor neuron disease showing FTLD-TDP type 3 pathology (sensu Mackenzie). Neuropathology 31(3):271-279

65. Kelley BJ, Haidar W, Boeve BF, Baker M, Shiung M, Knopman DS et al (2010) Alzheimer disease-like phenotype associated with the c.154delA mutation in progranulin. Arch Neurol 67(2): 171-7.

66. Kobayashi Z, Tsuchiya K, Arai T, Yokota O, Yoshida M, Shimomura Y et al (2010) Clinicopathological characteristics of FTLD-TDP showing corticospinal tract degeneration but lacking lower motor neuron loss. J Neurol Sci 298(1-2):70-77

67. Kuwahara H, Tsuchiya K, Saito Y, Kobayashi Z, Miyazaki H, Izumiyama $Y$ et al (2010) Frontotemporal lobar degeneration with motor neuron disease showing severe and circumscribed atrophy of anterior temporal lobes. J Neurol Sci 297(1-2):92-96

68. Kelley BJ, Haidar W, Boeve BF, Baker M, Graff-Radford NR, Krefft T et al (2009) Prominent phenotypic variability associated with mutations in Progranulin. Neurobiol Aging 30(5):739-751

69. Yoshida M, Kono K, Nomura M, Hashizume Y (2009) An autopsy case with semantic dementia, coexistence TDP-43-positive FTLD-U and Alzheimer disease pathology. In: Japanese Society of Neuropathology_Abstracts of the 50th annual meeting. Elsevier, Takamatsu, P 370.

70. Davion S, Johnson N, Weintraub S, Mesulam MM, Engberg A, Mishra M et al (2007) Clinicopathologic correlation in PGRN mutations. Neurology 69(11):1113-1121

71. Davies RR, Hodges JR, Kril JJ, Patterson K, Halliday GM, Xuereb JH (2005) The pathological basis of semantic dementia. Brain 128(Pt 9):1984-1995

\section{Publisher's Note}

Springer Nature remains neutral with regard to jurisdictional claims in published maps and institutional affiliations.
Ready to submit your research? Choose BMC and benefit from:

- fast, convenient online submission

- thorough peer review by experienced researchers in your field

- rapid publication on acceptance

- support for research data, including large and complex data types

- gold Open Access which fosters wider collaboration and increased citations

- maximum visibility for your research: over 100M website views per year

At BMC, research is always in progress.

Learn more biomedcentral.com/submissions 